\title{
What sports medicine practitioners should know about imaging for femoro-patellar pathologies
}

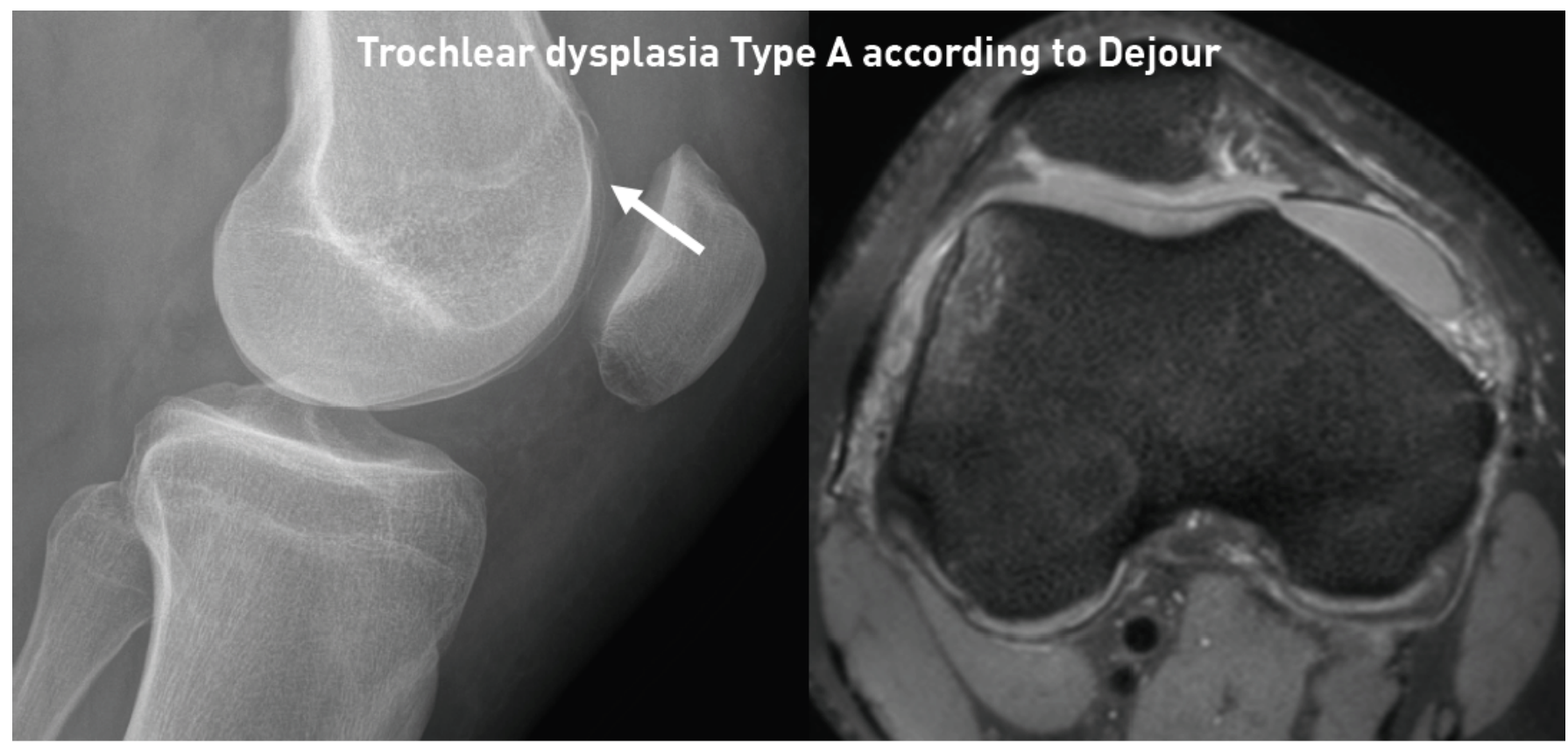

Hamard $\mathbf{M}^{1}$, Boudabbous $\mathrm{S}^{1}$, Tscholl $\mathbf{P M}^{2}$

${ }^{1}$ Departement of Radiology, University Hospitals Geneva, Geneva, Switzerland

${ }^{2}$ Department of Orthopedic and Trauma Surgery, University Hospitals Geneva, Geneva, Switzerland

\begin{abstract}
Clinical imaging in patellofemoral disorders is fundamental for the understanding of the pathology, and to find the adequate treatment option. Anatomical risk factors such as trochlear dysplasia, patella alta, lateralized tibiale tubercle (measured by the tibiale tubercle trochlear groove distance), torsional or coronal lower limb alignment that are the origine of patellar maltracking or even patellar dislocation, can be assessed with high reliability on conventional radiographs combined with MR imaging. The conservative and surgical treatment will highly rely on these findings. The importance of clinical imaging in unspecific anterior knee pain is less clear. It is mostly performed to exclude structural injuries, however is rarely reliable to diagnose the painful structure. Critical appraisal of morphological risk factors is necessary especially in the treatment of anterior knee pain, since fortuitous pathological findings are frequent.
\end{abstract}




\section{Résumé}

Une connaissance clinique adéquate est nécessaire pour une prise en charge optimale du syndrome fémoro-patellaire et pour l'interprétation de l'imagerie. Les anomalies anatomiques qui favorisent ce syndrome sont de nos jours bien investiguées par le couple radiographie conventionnelle-IRM. Ces anomalies sont nombreuses incluant la dysplasie trochléaire, la patella alta, une latéralisation du tubercule tibial (mesurée par la distance dite TAGT), anomalie d'alignement frontal ou de torsion des membres inférieurs; toutes ces anomalies sont à l'origine d'une mobilité inadéquate de la patella voir une luxation. Le traitement pourra être conservateur ou chirurgical et dépendra étroitement des trouvailles cliniques et radiologiques. En dehors de cette pathologie, le rôle clinco-radiologique est mal défini pour toute douleur antérieure du genou. Il a pour objectif principal d'éliminer d'éliminer des anomalies structurelles sans toujours trouver une corrélation avec la douleur. Une analyse critique et synthétique est nécessaire aussi bien pour les anomalies anatomiques trouvées ainsi que pour le choix du traitement.

\section{Introduction}

Anterior knee pain (AKP) is one of the most common complaints in athletes, especially performing pivoting and jumping sports. Many structures around the patella can become symptomatic, mainly by structural overuse with or without patellar maltracking, poor muscle control or functional instability of the lower limb. The most frequently painful structures are the medial and lateral patellofemoral retinacula, the entheses of the patellar and less frequently the quadriceps tendon, and the intra-articular but extrasynovial fat pads. These structures, although visible on clinical imaging, might not always appear pathologic if painful, however are frequently associated with morphological risk factors. The analysis of the frequently painful structures and the anatomy predisposing to patellar maltracking requires a systematic approach on multi-modal imaging by radiologists and clinicians for the best understanding of the underlying causes of anterior knee pain.

The indication, advantages and disadvantages of the different imaging modalities will be here discussed in the context of anterior knee pain.

\section{Patellofemoral dysplasia - radiological signs and measurements}

\section{Conventional radiography (CR)}

$\mathrm{CR}$ allows detection and a rapid and global appreciation of the bony structure of the knee, the morphology of the patellofemoral joint and subchondral degenerative changes. The evaluation of AKP requires a weight-bearing antero-posterior (AP) view to evaluate the femoro-tibial knee joint, a strict lateral (L) view with overlapping posterior medial and lateral femoral condyles and an axial imaging in $30^{\circ}$ of knee flexion (Table 1). 


\begin{tabular}{|c|c|c|c|}
\hline CR & Measurements & Further information & Remarks \\
\hline Lateral view & $\begin{array}{l}\text { Trochlear dysplasia [Dejour] } \\
\text { Patella alta [CD-I/InSa-I] }\end{array}$ & $\begin{array}{l}\text { Loose bodies } \\
\text { Osteophytes, effusion } \\
\text { Patella bipartita }\end{array}$ & $\begin{array}{l}\text { True lateral imaging absolutely } \\
\text { mandatory }\end{array}$ \\
\hline Axial view & Wiberg classification & $\begin{array}{l}\text { Osteophytes } \\
\text { Osteochondral fractures } \\
\text { Inferomedial patellar spur }\end{array}$ & $\begin{array}{l}\text { No information about trochlear } \\
\text { dysplasia }\end{array}$ \\
\hline AP view/long axis & Limb alignment & $\begin{array}{l}\text { Femoro-tibial status } \\
\text { Patella bipartita }\end{array}$ & \\
\hline
\end{tabular}

\begin{tabular}{|c|c|c|c|}
\hline \multicolumn{4}{|l|}{ MRI } \\
\hline Sagittal plane & $\begin{array}{l}\text { Patellar height (CD-I, InSa-I, } \\
\text { PT-I) }\end{array}$ & $\begin{array}{l}\text { Osteochondral fragments } \\
\text { Superolateral Hoffa impingements } \\
\text { Patellar and quadricipital } \\
\text { tendinopathy }\end{array}$ & $\begin{array}{l}\text { Patellar height might be difficult } \\
\text { to analyse if patella subluxated }\end{array}$ \\
\hline Axial plane & $\begin{array}{l}\text { Trochlear Dysplasia (Dejour) } \\
\text { Lateral trochlear inclination } \\
\text { angle } \\
\text { Sulcus angle, patellar tilt, } \\
\text { trochlear depth, trochlear } \\
\text { facet asymmetry } \\
\text { TT-TG and PT-TG } \\
\text { Femoral and tibial torsion }\end{array}$ & $\begin{array}{l}\text { Cartilage injuries } \\
\text { Osteochondral fragments } \\
\text { MPFL injury } \\
\text { Bone bruise } \\
\text { Plica mediopatellaris } \\
\text { Patella bipartita }\end{array}$ & $\begin{array}{l}\text { Highly dependent on height } \\
\text { selection of the axial imaging }\end{array}$ \\
\hline
\end{tabular}

Table 1: Imaging modalities, measurements and practical hints for investigation of patellofemoral disorders

The most important morphological risk factors for patellar maltracking and patellar dislocation are based on the studies by Henry [1,2] and David Dejour [3] and are measured on the strict L view (trochlear dysplasia and patella alta). The tibial tubercle trochlear groove distance (TT-TG), initially measured on axial CR [4] is currently exclusively measured on multi-slice imaging (CT or MRI).

Trochlear dysplasia has according to Dejour et al [3] three radiographic features that are associated with a flat or even convex trochlea: the crossing sign (when the line of the trochlear groove crosses the highest point of the lateral trochlear facet), the double contour (when there is hypoplasia of the medial femoral condyle and the medial trochlear facet) and the supratrochlear spur (Figure 1). Only strict L view permit to evaluate this classification. Important to note, that trochlear dysplasia cannot be diagnosed on axial CR [5]. Patella alta is the second main morphological risk factor which can be visualized on the $L$ view. Several indices have been proposed to evaluate the position of the patella, whereby the Insall-Savati (InSa-I) [6] and Caton-Deschamps index (CD-I) [7] are the most frequently used on CR, CT and MRI (Figure 2A) [8]. Patella alta is defined by a InSa-I of $>1.3-1.4$ or a CD-I by $>1.3$, although the cut-off might vary in literature. Furthermore, surgical correction might be indicated only if CD-I is above 1.4, hence relativizing the cut-off values of patella alta [8].

Patellar dysplasia can be appreciated on axial CR according to the Wiberg classification. Patellae with a very short medial and therefore vertical facet (Wiberg type 3) are associated with trochlear dysplasia. This classification, however, has only limited clinical relevance [9]. An infero-medial patellar spur is highly associated with trochlear dysplasia and can be a sign of patellar instability (Figure 3) [10]. 
Long leg standing CR can be indicated to evaluate associated valgus, which has been found to be an additional risk factor for patellar instability recently [11].
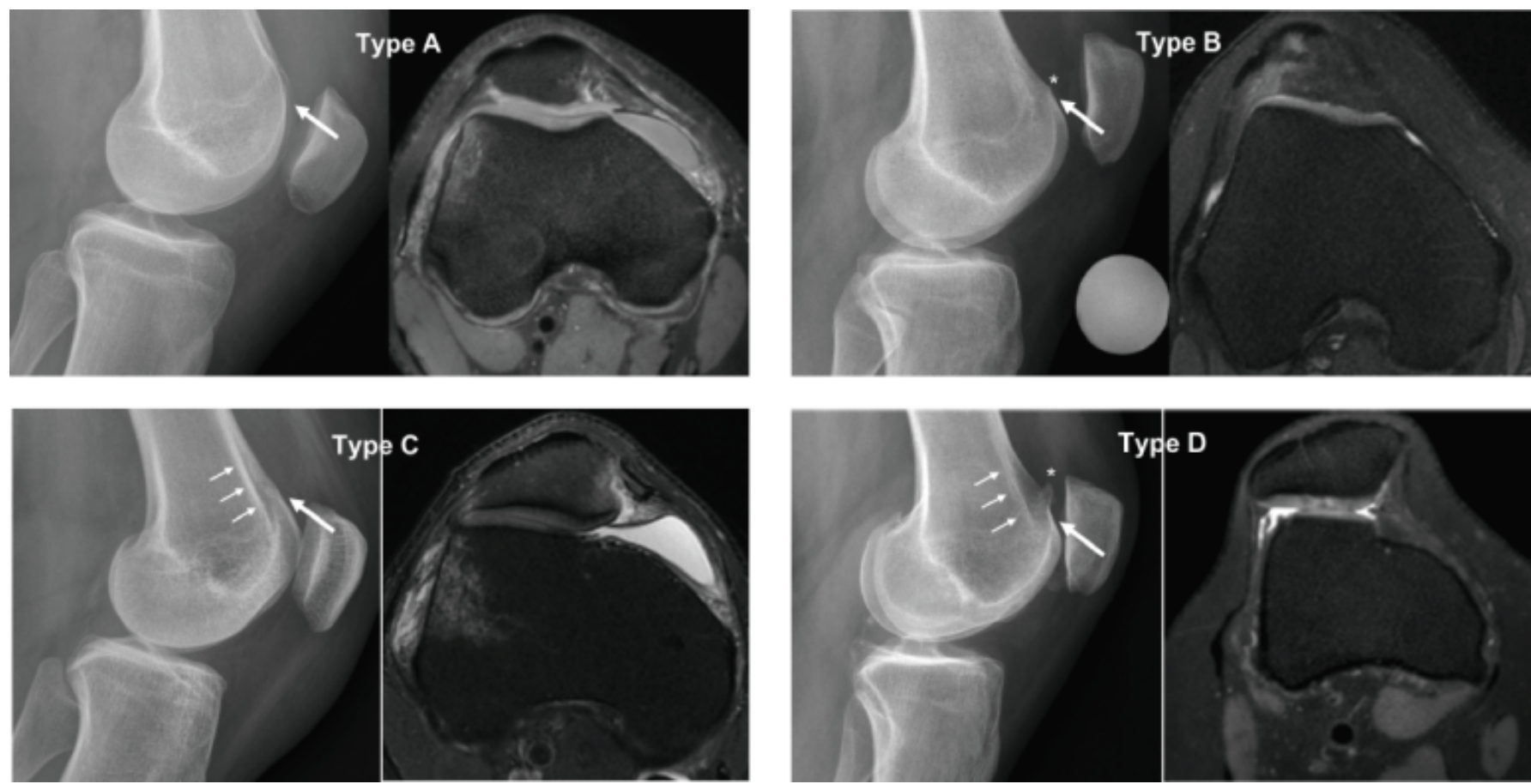

Figure 1: Trochlear dysplasia type A to type D according to David Dejour (1998) on strict L CR and axial multislice imaging. The combination of the three radiographic signs on CR permits to classify trochlear dysplasia: crossing sign (white arrow), supratrochlear spur (white asterix) and double contour (small white arrows). On multi-slice imaging, the trochlear groove is shallow (type A), flat (type B), convex (type C) or shows the «cliff-pattern» (type D). 

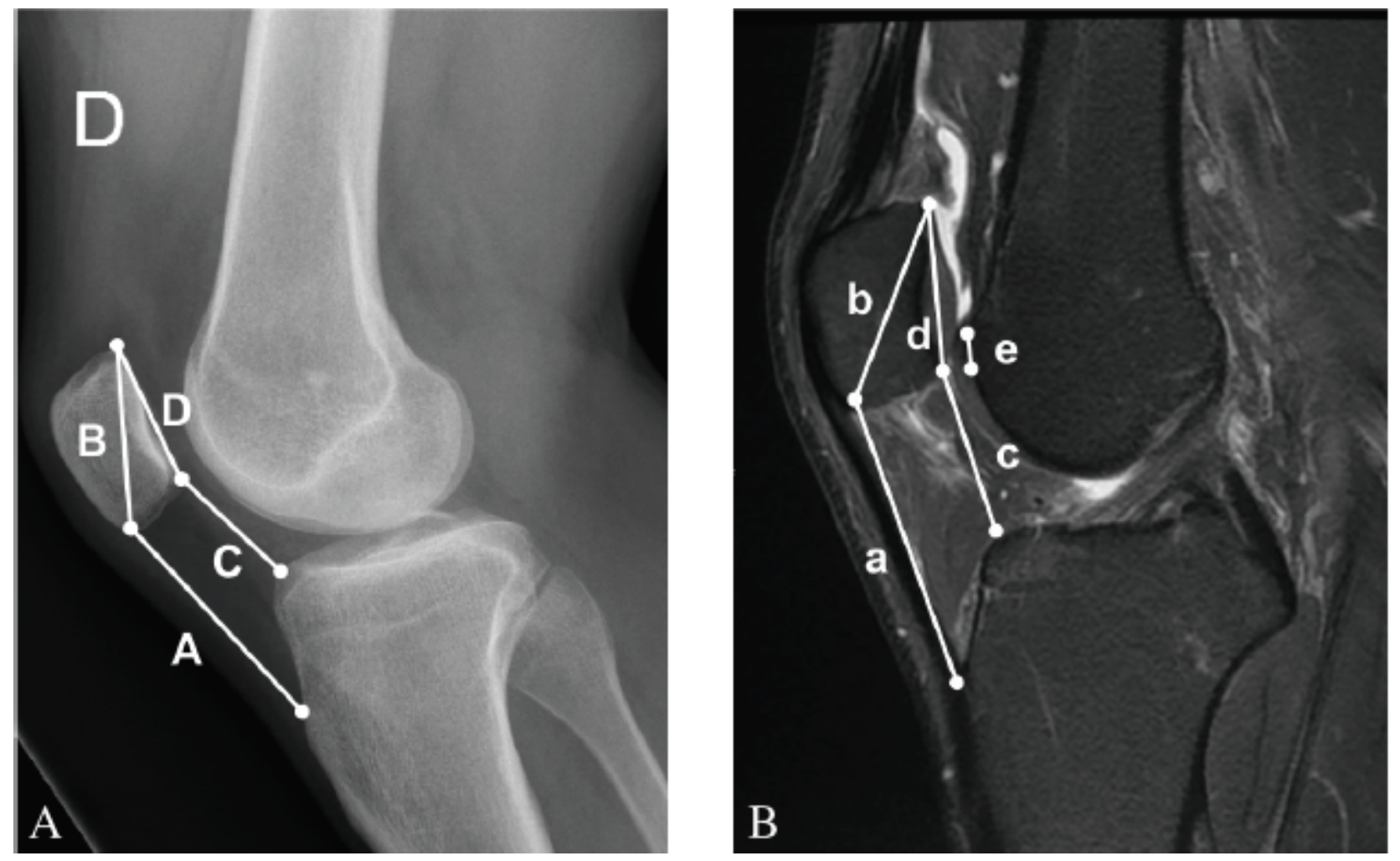

Figure 2: Patellar height measurement on strict L CR view and sagittal MRI. Insall-Salvati Index = A:B; CatonDeschamps Index = C:D; Patello-Trochlear Index = e:d

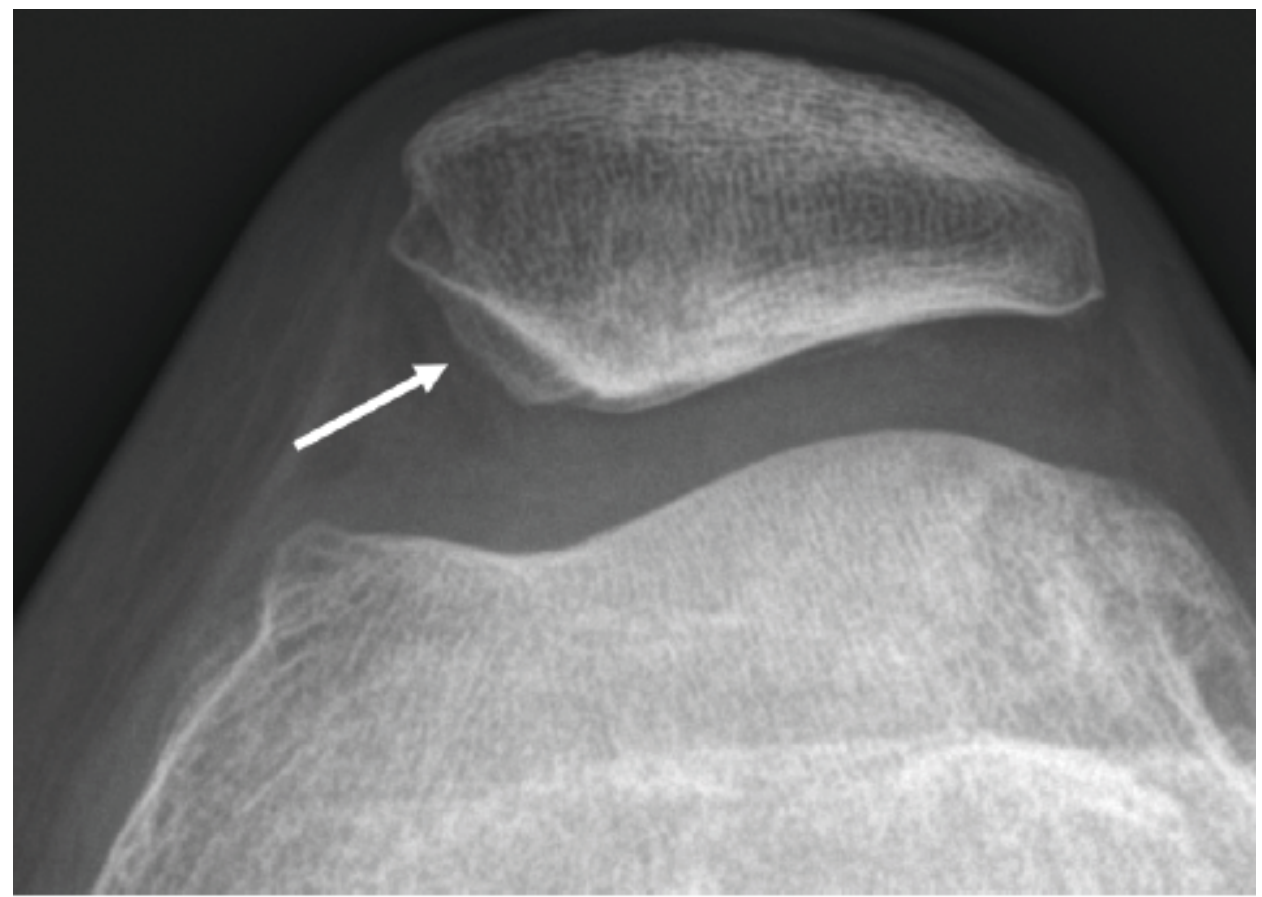

Figure 3: Inferomedial patellar spur - a sign of distal patellar dysplasia on axial $\mathrm{CR}$, highly associated with trochlear dysplasia and patellar instability. 


\section{Multi-slices imaging modalities (MRI/CT)}

Computed tomography (CT) and magnetic resonance imaging (MRI) are both nowadays widely available and permit multiplanar reconstructions in every planes. Whereas CT scan acquisition is much faster, irradiation has been minimized using cone-beam СТ (СВCT) and the resolution of bony structure is more accurate, there is no irradiation with MRI and cartilage and soft-tissue are better visualized. MRI therefore is the gold-standard in patellofemoral assessment, especially in the early evaluation after primary patellar dislocation.

Whereas both imaging modalities can detect the main predisposing factors for patellar instability, cut-off values may differ due to the different landmarks (bony on CT versus cartilaginous-tendinous landmarks on MRI for trochlear morphology) and to the different flexion angle of the knee during acquisition of the images. Whereas CT are always performed in full extension, MRI are performed at about $10-15^{\circ}$ of knee flexion due to the position in the knee coil, highly influencing the TT-TG value or patellar tilt. Measuring trochlear dysplasia on 2D imaging has been introduced by David Dejour in 1998 on CT scan [3], and has led to the four type classification, from type A having a shallow trochlea to type D with a convex trochlea and the cliff pattern due to the hypoplastic medial trochlea and the absent trochlear groove (Figure 1). The classification of trochlear dysplasia on CT and MRI might differ due to the different osseous and cartilaginous trochlear surface, and also according the level of the axial image. Therefore, the trochlea needs to be assessed from proximal to distal for complete appreciation of its morphology and not solely on a single cut [12]. There are several more measurement methods on axial imaging, such as the lateral trochlear inclination angle [13], sulcus angle [14], trochlear depth [14] and trochlear facet/condyle asymmetry (14) all able to describe trochlear dysplasia, however with only limited clinical relevance. The most common patellar height ratios used on CR (InSa-I and CD-I) can also be measured on MRI (Figure 2B), however require a marginal correction of 0.1 for InSa-I and CD-I according to a comparative CR/CT/MRI study $[15,16]$. However, it might be difficult to find a single sagittal cut on CT or MRI which represents the entire patellar tendon and the patella, making the measurement of the InSa-I sometimes impossible [8]. More recently, the ratio of the overlap between the patellar and the trochlear cartilage has been proposed by Biedert [17] and slightly modified by others [18,19]. This type of ratio however is highly depending on knee flexion angle, and on patellar position and needs therefore to be analysed carefully. The patellar tendon length with a cut-off value of $52 \mathrm{~mm}$ has gained only little attention in literature, probably due to the high interpersonal variability.

The tibial tubercle to trochlear groove (TT-TG) distance is a measurement for a distal lateral patellar vector force and represents the distance between the trochlear groove and the tibial tubercle and is increased above $15 \mathrm{~mm}$ and generally associated with trochlear dysplasia if above $20 \mathrm{~mm}$ [20]. It can nicely be measured on CT by superposing two axial slices (Figure 4) or measured with adequate tools on MRI. Improved inter-rater reliability has been shown using the centre of the patellar tendon on the tibial tubercle (PT-TG). Some difficulties may arise in high-grade trochlear dysplasia where the trochlear groove is found only distally. Due to the distally lateral orientation of the trochlear groove, the TT-TG will be underestimated in these cases [21]. The measurement results of CT and MRI cannot be compared. The flexed knee position on MRI decreases the TT-TG significantly compared to the fully extended knee on CT which undergoes the "screw-home mechanism" close to extension (Figure 5A and B). Therefore, TT-TG might vary up to 8-10 $\mathrm{mm}$ between CT and MRI. Due to the dynamic susceptibility of the TT-TG in full extension and the improved interrater reliability using soft-tissue landmarks, MRI in a standard knee coil 
should be preferred.

The patellar tilt, the lateral decline of the patella, described by Dejour [2] as one of the 4 major risk factors and pathologic if $>20^{\circ}$, is thought to be rather a consequence of trochlear dysplasia, patellar height, the TTTG and probably also the patellar shape than an independent risk factor for patellar instability, and has only limited clinical evidence as a single measurement [22].

The femoral torsion is a further risk factor for patellar instability, which can be measured only on CT and MRI. It stands for the angle of the femoral neck to the posterior condylar line of the distal femur (Figure 6). For the MRI, the knee coil has to be removed, and the imaging is performed in full extension of the knee. The exact measurement and their cut-off values especially for surgical correction remain highly debated in literature [23], because of the wide range of values seen in healthy patients [24] and its several measurement techniques [25-28]. Usually, excessive femoral anterior torsion is considered $>25^{\circ}$. Since clinical examination may not be able to evaluate femoral torsion consistently, femoral and tibial torsion measurements on MRI (or CT) should be standard procedure for the assessment of patellofemoral instability.

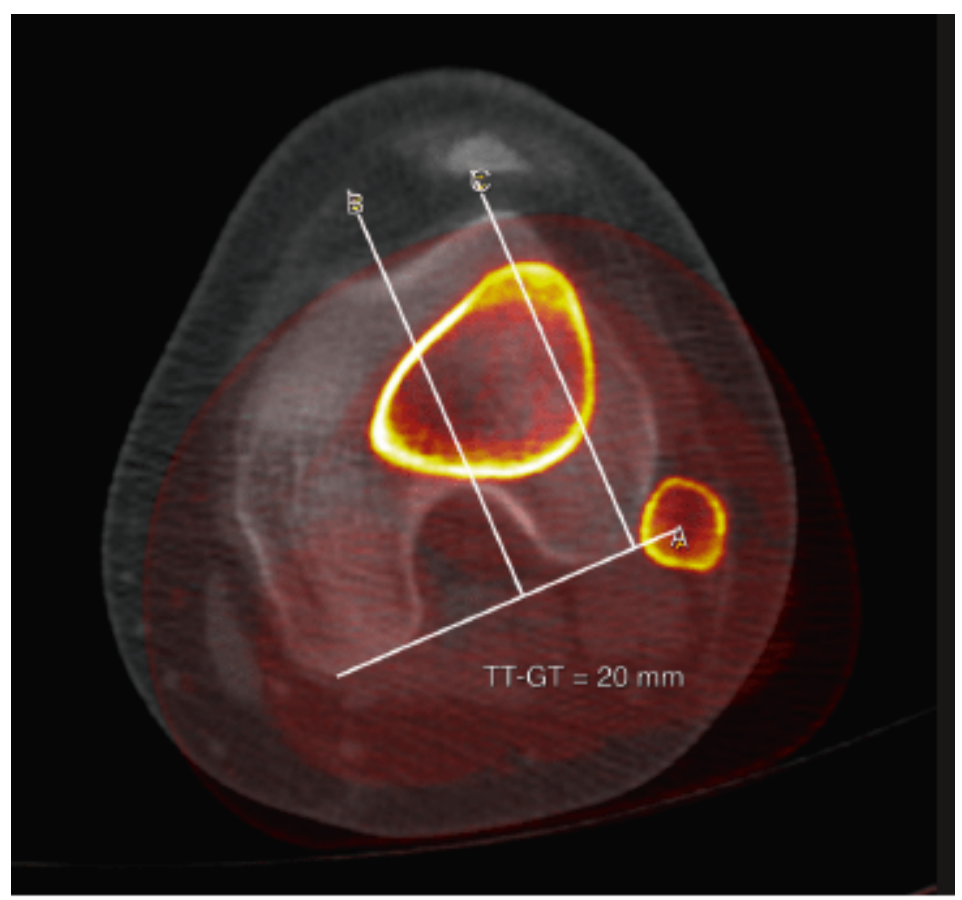

Figure 4: TT-GT distance on CT-scan. The distance between the trochlear groove and the tip of the tibial tubercle is measured along the posterior condylar line. 

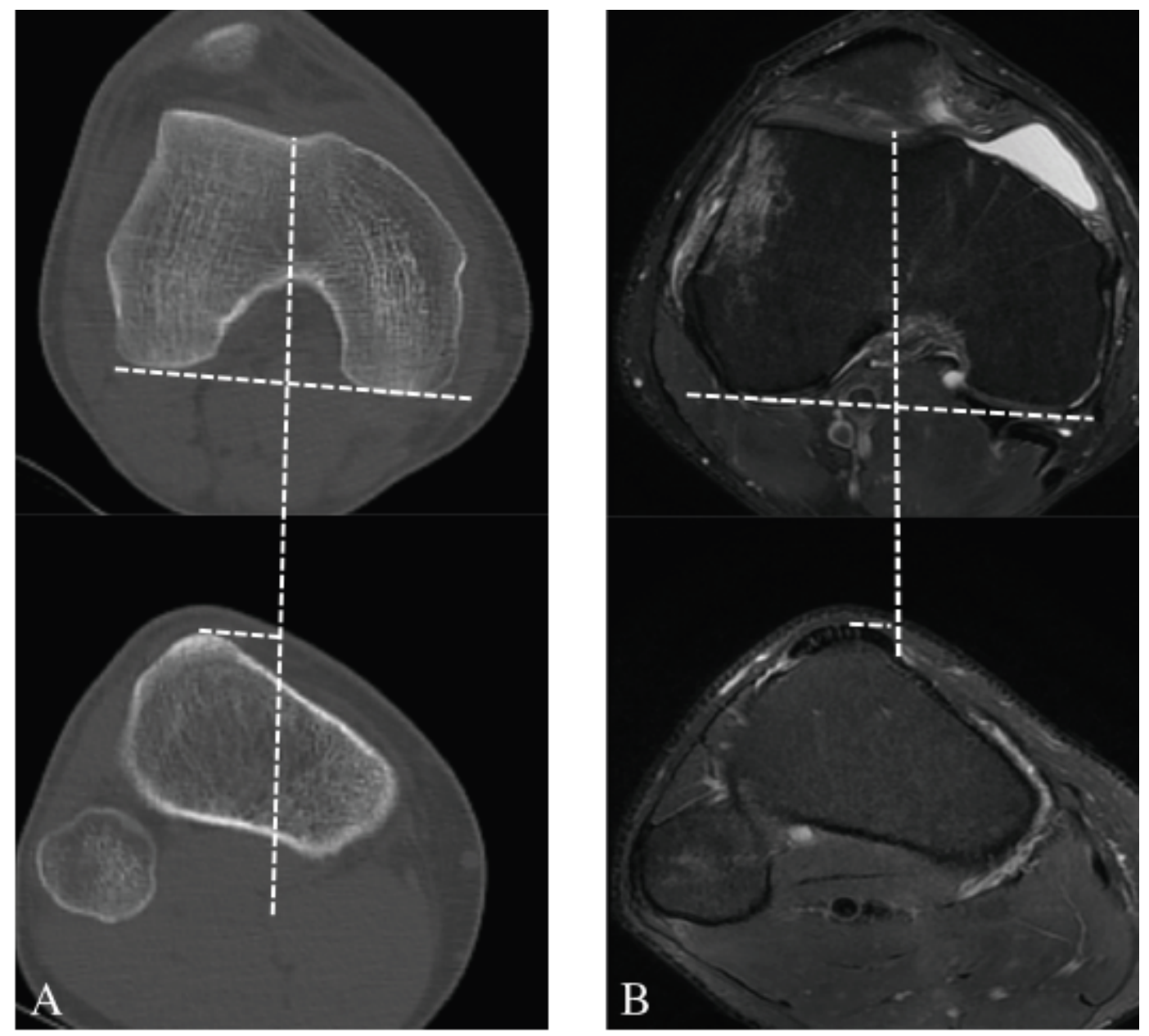

Figure 5: TT-TG distance on A) CT and B) MRI. Note the difference of bony and cartilaginous landmarks, as well as flexion angle influencing the TT-TG distance. 


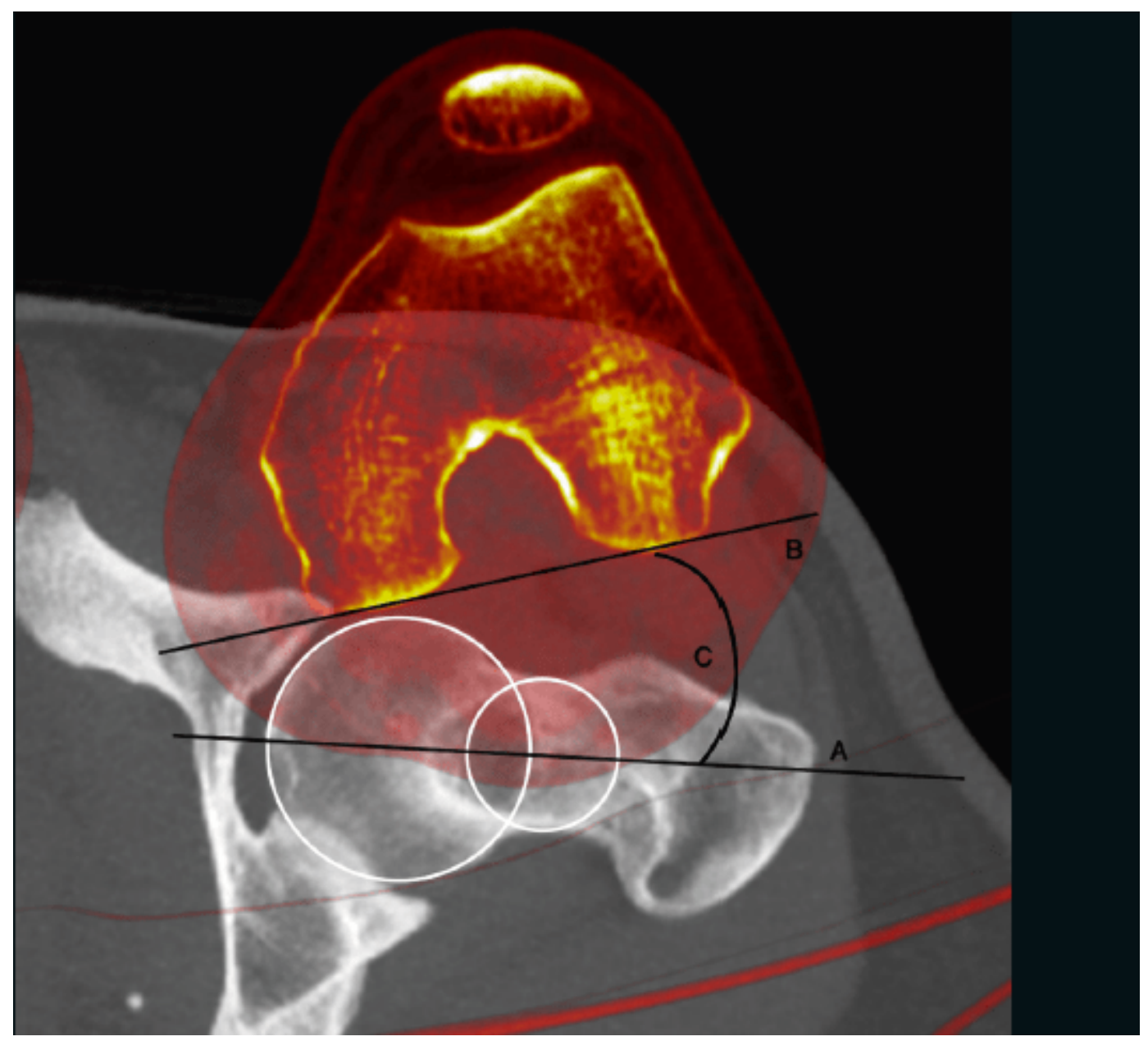

Figure 6: Femoral torsion measured on axial CT (or MRI). Line A is a line through the femoral neck and line B a tangent to the posterior femoral condyles. Its angle (c) results in the femoral antetorsion.

\section{Radiological signs of patellar dislocation}

Acute patellar dislocation can be diagnosed on CR if the patella is locked, which is rare, or after reduction with a osteochondral fracture seen in the parapatellar recessus or a defect in the medial patellar facet on the axial CR view. A positive Holmgren sign, with a clearly delimited level showing liphemarthrosis, can be visible as well on lateral view, showing osseous injury. CT scan may show additionally condensed bone zones on the lateral femoral condyle and the inferomedial patella due to the impaction of the lateral patellar dislocation. MRI is able to show this bone bruise for several month, can visualize the entire size of the osteochondral lesion - which is almost exclusively seen in first episode of patellar dislocation (12-15\%) - and MPFL rupture/tear which is present in almost all patients after primary patellar dislocation (Figure 7). In recurrent patellar dislocation, signs of MPFL rupture may be absent. Associated injuries occur are rarely seen, such as anterior cruciate ligament injuries, or medical collateral ligament tear. 

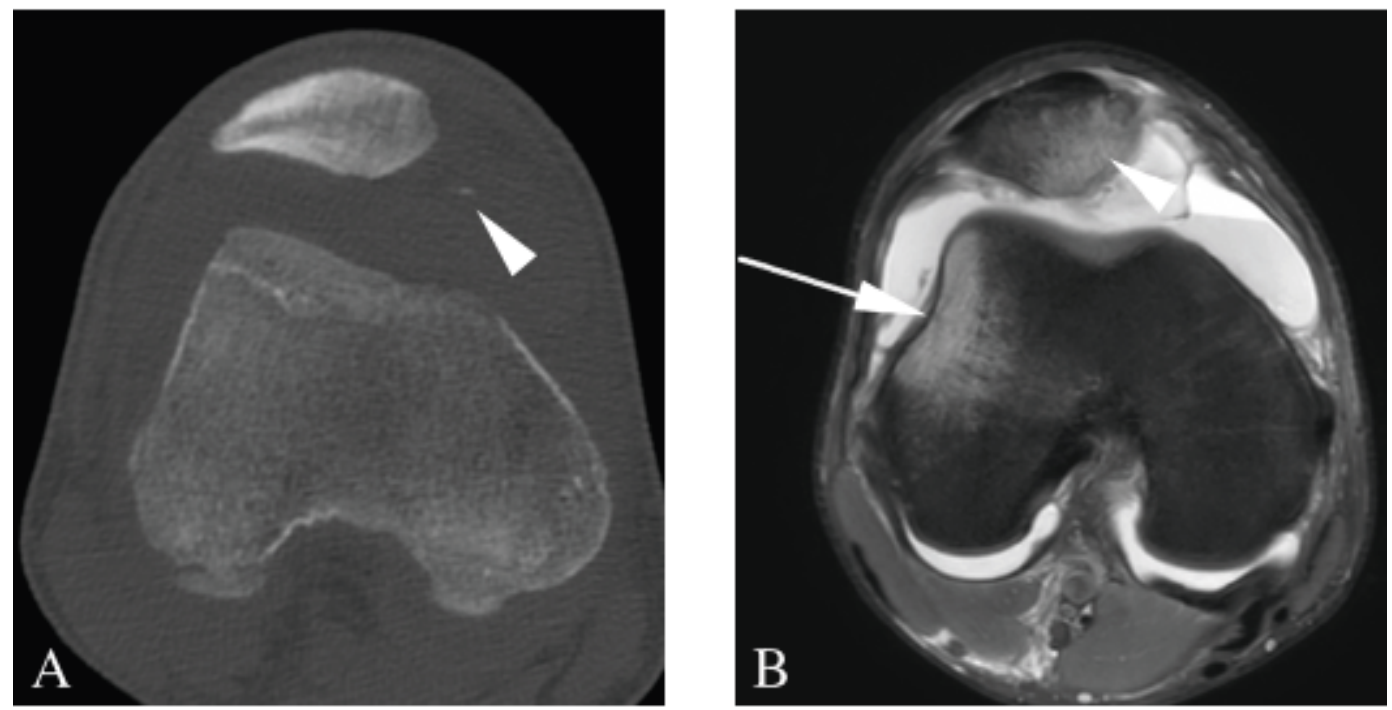

Figure 7: Signs of patellar dislocation on A) axial CT and B) axial MRI. A) Osteochondral

fragment (arrow head on CT) from the inferomedial patellar facet, B) with the

pathognomonic bone bruise on the lateral femoral condyle (white arrow) and the medial patellar border (arrow head on MRI). The Holmgren sign represents liphemarthrosis, that can be visible also on L CR.

\section{Imaging findings in anterior knee pain and associated pathologies}

The painful structures in anterior knee pain are almost exclusively the soft-tissues. The MRI (and ultrasonography) is therefore the imaging of choice:

Jumper's knee, is an exercise related painful condition most frequently located at the distal tip of the patella. It mainly affects young athletes involved in jumping sports, that either perform a structural over use or a relative overuse in combination with either patella alta, or mild trochlear dysplasia [29]. Most frequently, CR and MR imaging shows no pathologic findings. However, in the case of degenerative tendinopathy, the best imaging modality to assess this pathology is the MRI, which highlights proximal thickening of the patellar tendon of $>7 \mathrm{~mm}$, or intra-tendinous signal abnormalities near its central or medial insertion to the patella in chronic cases (Figure 8). [29] In only few cases, this tendinopathy affects the superficial fibers that stretch from the patellar tendon directly to the quadriceps tendon (galea aponeurotica).

The superolateral impingement syndrome is characterized by a friction of the infrapatellar and lateral fat pad between posterior side of patellar tendon and the anterior side of lateral femoral condyle [30,31]. It seems to be associated with patella alta, genu valgum or an excessive TT-TG. On MRI, oedema of the superolateral Hoffa fat pad and focal patellar tendinopathy can be seen (Figure 9). Due to the high-riding and often tilted patella, associated cartilage injury of the lateral trochlear and patellar facet are not unfrequent.

The pre-femoral fat pad impingement syndrome is caused by irritation of the pre-femoral fat pad that lies anterior to the distal femur, between the subquadricipital recessus and the anterior cortex of the distal femur. It is often associated with patella alta and femoropatellar maltracking (Figure 10). MRI shows oedema in the prefemoral fat pad in his central or inferolateral part. The mediopatellar plica syndrome is usually presented as a diffuse anterior knee pain, with or without 
clicking or sometimes even a sensation of locking. It only can be visualized on MRI as a thin plicature of the capsule medial to the patella (Figure 11). There is no sign on imaging that proofs a conflict. Patient's history, clinical imaging and its size lead to the diagnosis. Consecutive cartilage damage of the medial patellar facet is rare. The superopatellar plica or the intercondylar plica are other embryonic remnants, however much more rarely symptomatic.

Degenerative cartilage lesions are very frequently seen on MR imaging, most often however asymptomatic. Since there are no nerve endings in the cartilage, the origin of pain most probably lies in the subchondral bone. MR imaging (or in some selected cases where arthro-CT scan might be necessary) can graduate and localize the extent of the lesion according to the Outerbridge classification.

Patella bipartita describes the presence of an unfused ossicle usually found on the superolateral aspect of the patella (Figure 12), and can be found in 1-2\% of the population. It usually is asymptomatic, however direct trauma to the patella can induce pain at fibrous scar, which can be visible on MRI.
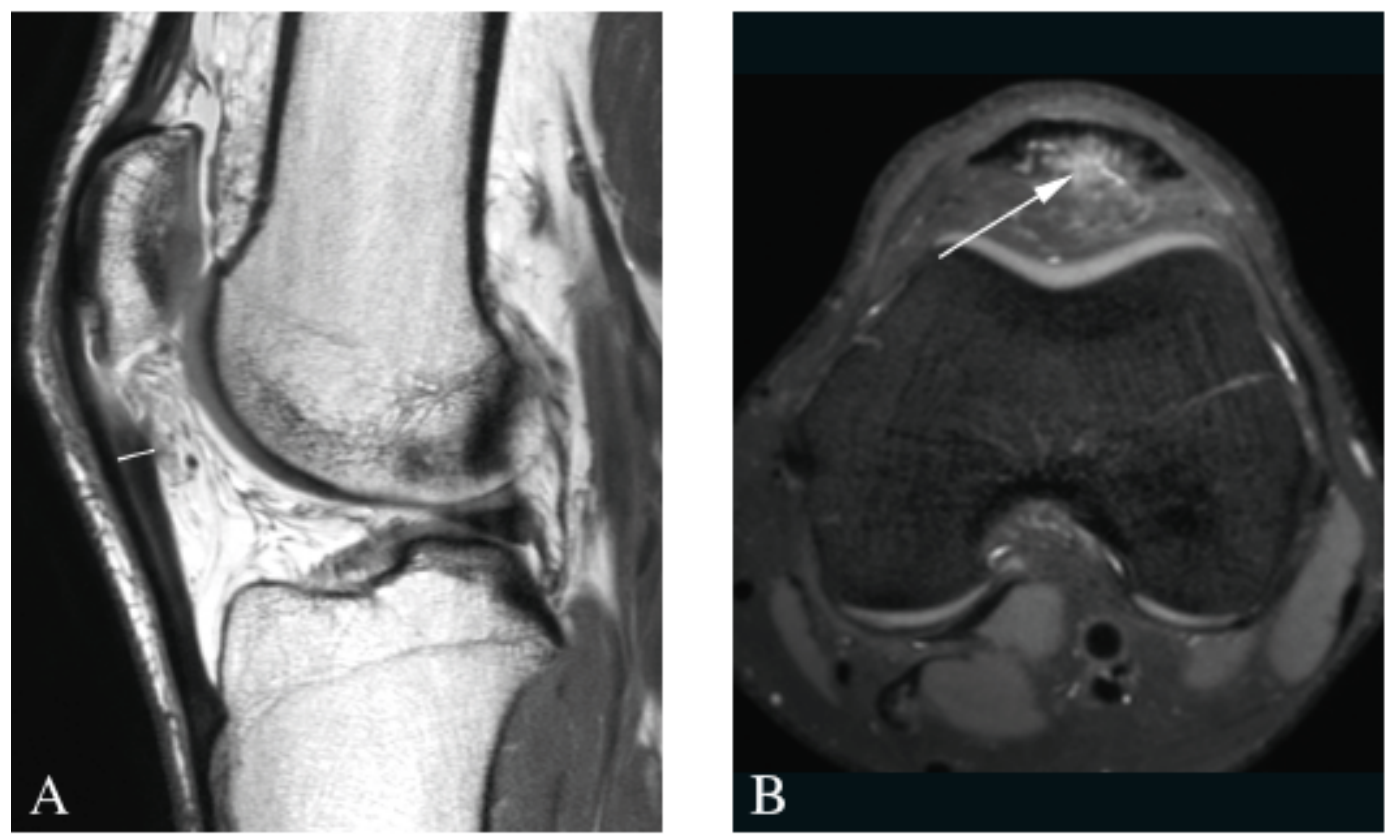

Figure 8: Jumper’s knee with structural intra-tendinous signal alterations on MRI (A) lateral plane PD, and B) axial plane PD fatsat). Thickening of the patellar tendon >7 mm (A; white line), and posterior signal abnormalities in the central or medial part of the tendon high signal T2w or PD images (B; white arrow). 

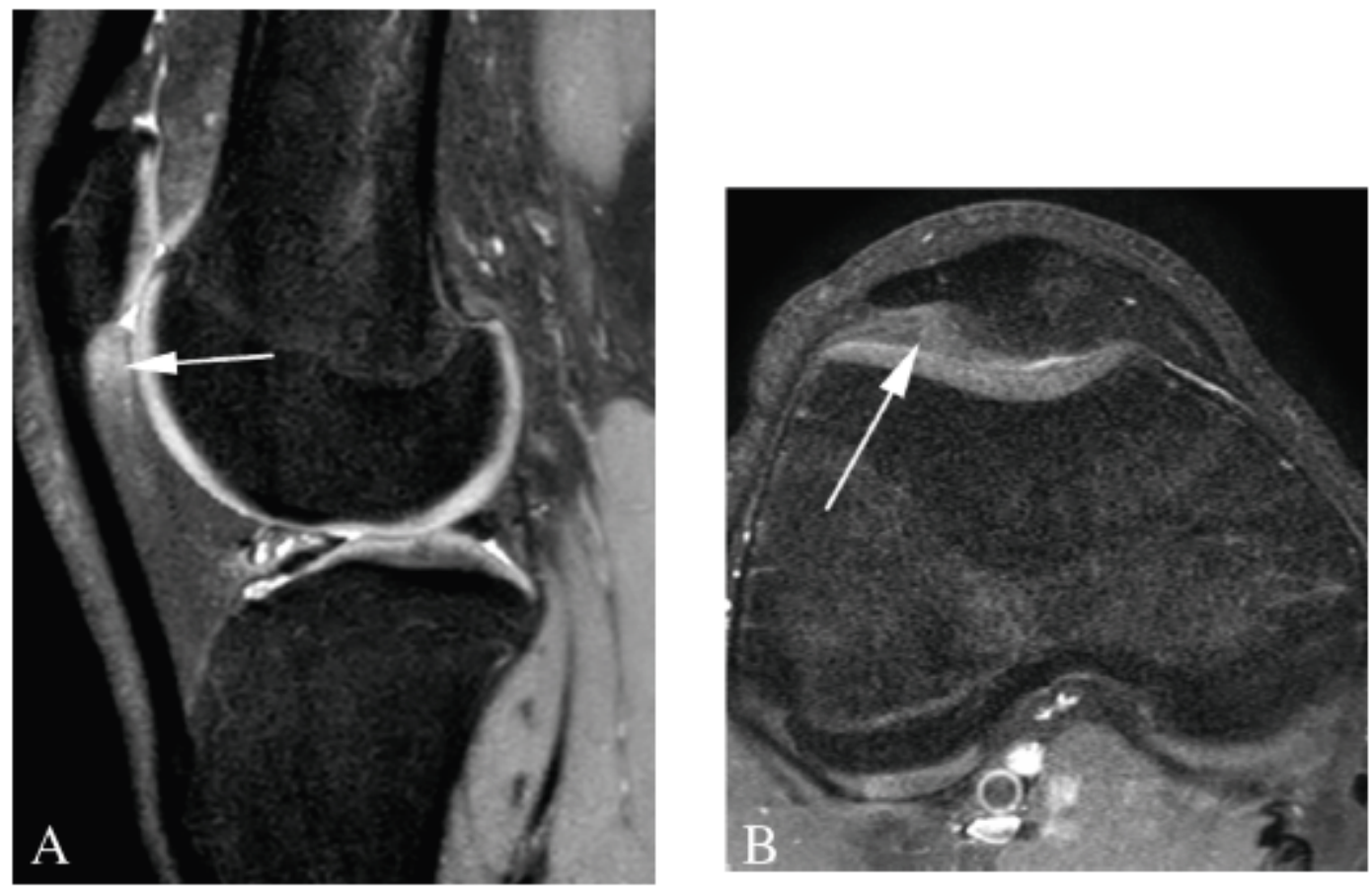

Figure 9: Superolateral Hoffa impingement on lateral and axial MRI (PD fat sat, white arrow) characterized by its lateral location, and patella alta. 

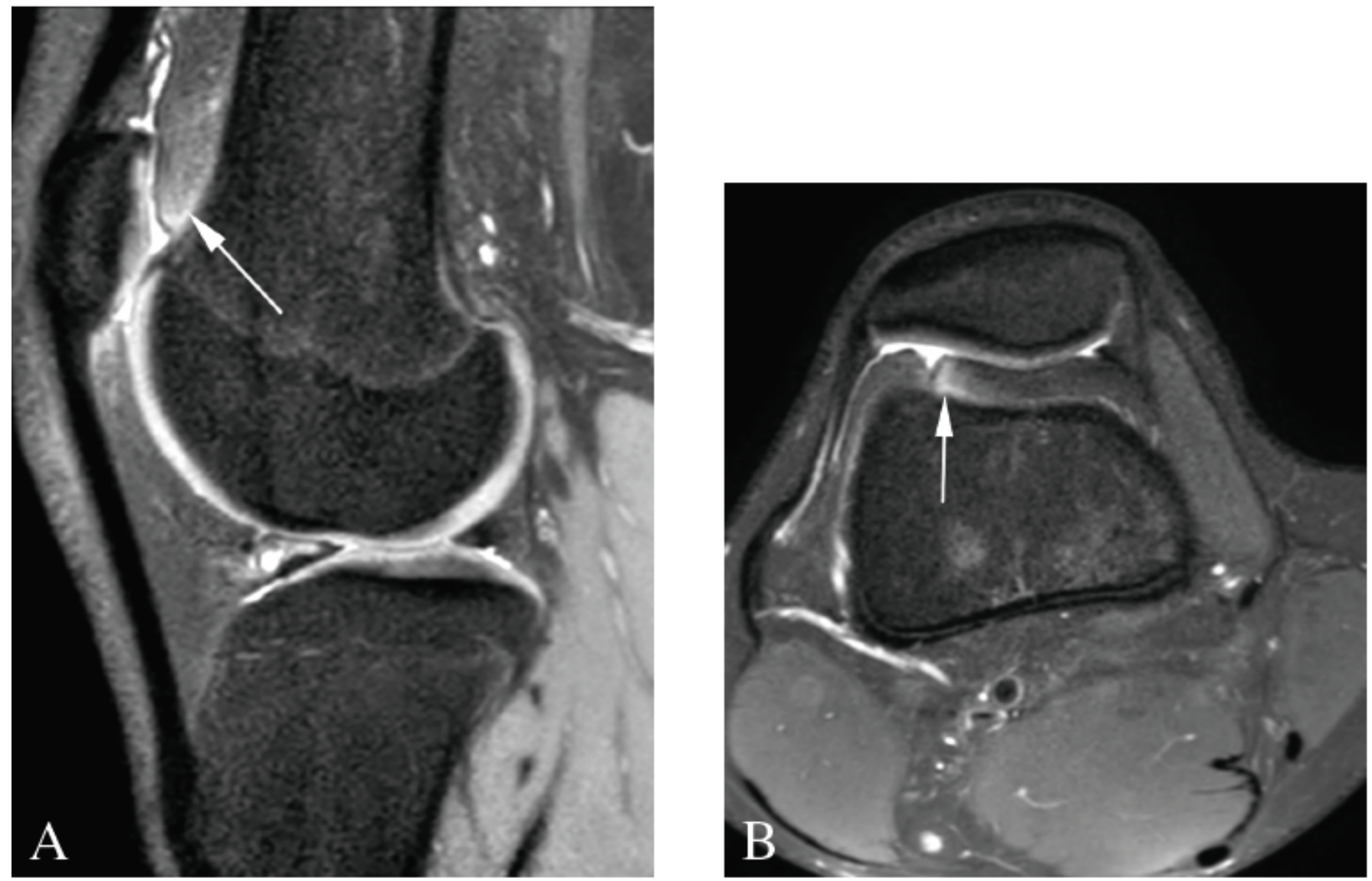

Figure 10: Pre-femoral fat pad impingement seen as hyperintensity on sagittal and axial MRI (PD fat sat). Its lateral localisation indicates lateral patellar dynamic subluxation. 


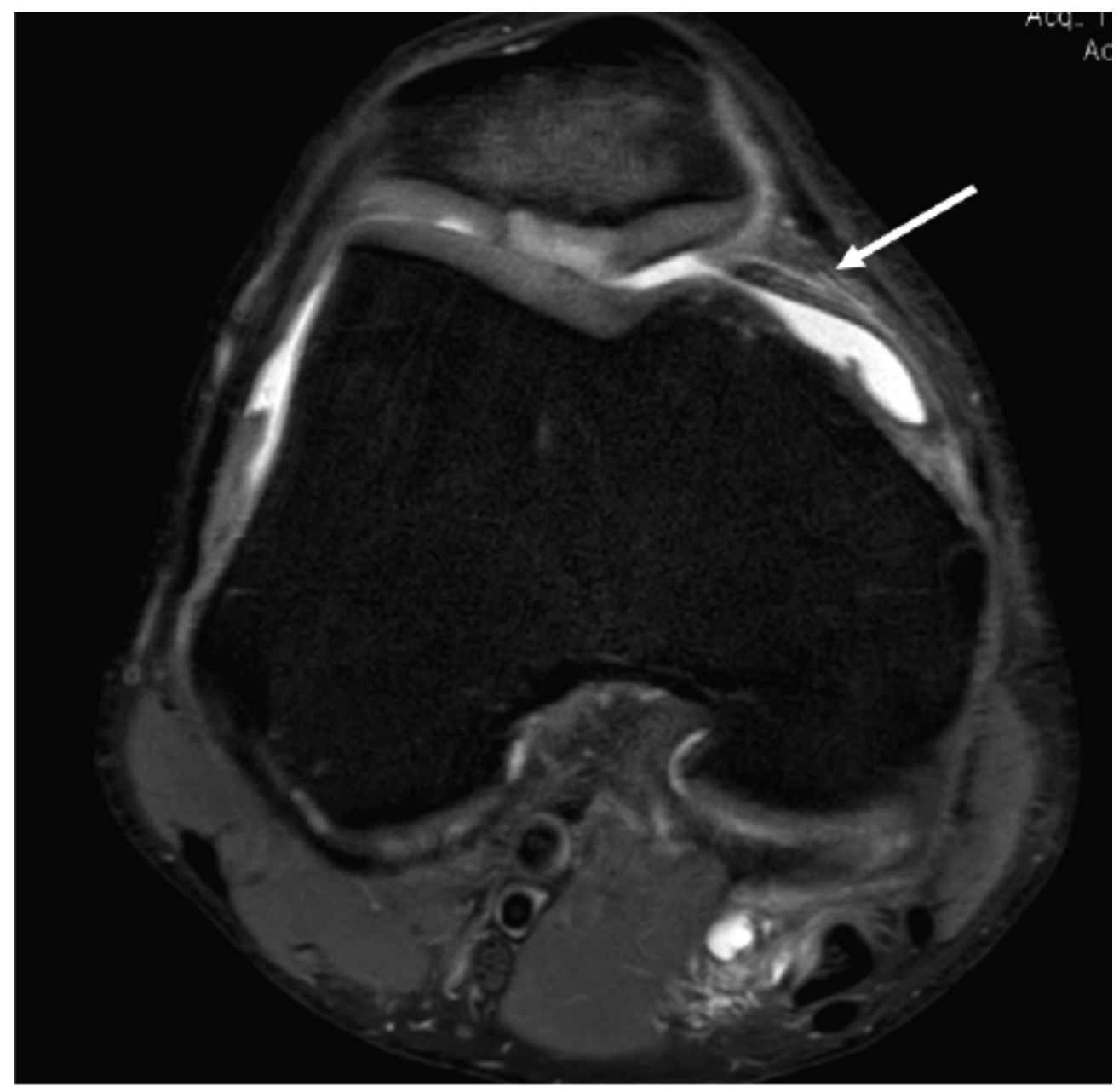

Figure 11: The mediopatellar plica seen as a thin plicature of the knee capsule (white arrow), in contact with the medial patellar facet (axial MRI, PD fat sat). 


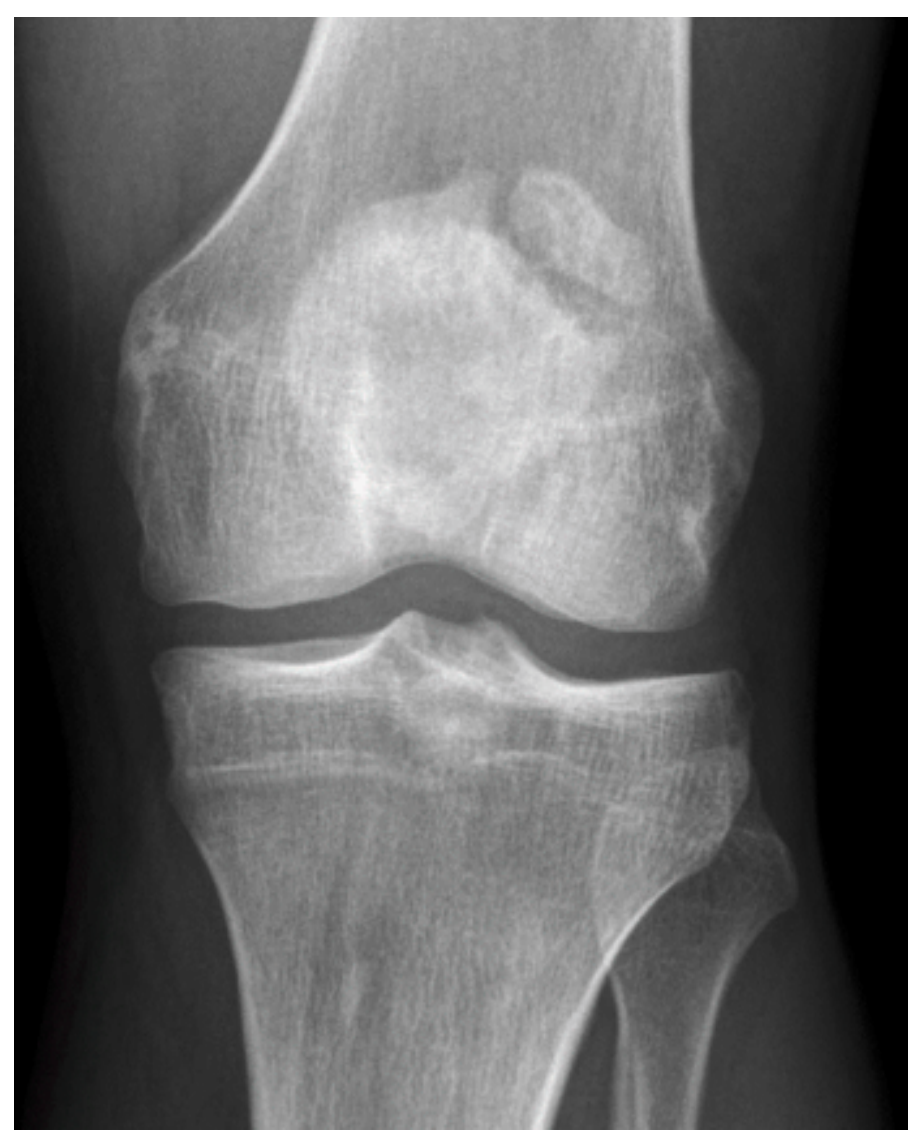

Figure 12: The non-fusioned ossicle of the patella bipartite most frequently is seen on the supero-lateral border of the patella.

\section{Practical implications}

- $\mathrm{CR}$ and MR imaging is mandatory to diagnose patellar dislocation, to visualize associated injuries and to evaluate patellofemoral anatomy known as the major risk factor for recurrent instability.

- Trochlear morphology, patellar height and torsional alignment also highly influences patellar tracking, that can be the origin of anterior knee pain.

- Its understanding in combination with clinical examination is the mainstay of a successful treatment.

\section{Corresponding author}

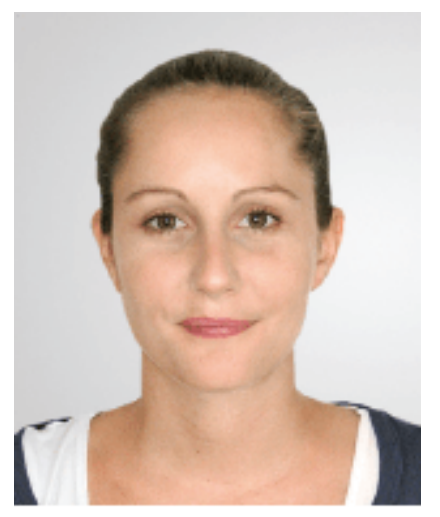


Département de Radiologie

Hôpitaux Universitaires Genève

Rue Gabrielle-Perret-Gentil 4

1205 Genève

marion.hamard@hcuge.ch $\mathrm{x}$

\section{Bibliography}

1. Dejour H, Walch G. [Chronic posterior instabilities]. Orthopade. 1987;16(2):149-56.

2. Dejour H, Walch G, Nove-Josserand L, Guier C. Factors of patellar instability: an anatomic radiographic study. Knee Surg Sports Traumatol Arthrosc. 1994;2(1):19-26.

3. Reynaud P, Dejour D. [Patellar entrapment due to a force-impaction fracture of the lateral condyle. Apropos of a case]. Rev Chir Orthop Reparatrice Appar Mot. 1998;84(8):752-5.

4. Goutallier D, Bernageau J, Lecudonnec B. [The measurement of the tibial tuberosity. Patella groove distanced technique and results (author's transl)]. Rev Chir Orthop Reparatrice Appar Mot. 1978;64(5):423-8.

5. Salzmann GM, Weber TS, Spang JT, Imhoff AB, Schottle PB. Comparison of native axial radiographs with axial MR imaging for determination of the trochlear morphology in patients with trochlear dysplasia. Arch Orthop Trauma Surg. 2010;130(3):335-40.

6. Hinckel BB, Gobbi RG, Kihara Filho EN, Demange MK, Pecora JR, Camanho GL. Patellar TendonTrochlear Groove Angle Measurement: A New Method for Patellofemoral Rotational Analyses. Orthop J Sports Med. 2015;3(9):2325967115601031.

7. Caton J, Deschamps G, Chambat P, Lerat JL, Dejour H. [Patella infera. Apropos of 128 cases]. Rev Chir Orthop Reparatrice Appar Mot. 1982;68(5):317-25.

8. Biedert RM, Tscholl PM. Patella Alta: A Comprehensive Review of Current Knowledge. Am J Orthop (Belle Mead NJ). 2017;46(6):290-300.

9. Otto A, Tscholl PM, Paasuke R, Herbst E, Willinger L, Imhoff AB, et al. Neither lateral patellar facet nor patellar size are altered in patellofemoral unstable patients: a comparative magnetic resonance imaging analysis. Knee Surg Sports Traumatol Arthrosc. 2019.

10. Saragaglia D, Mader R, Blaysat M, Mercier N. Medial facet patelloplasty in patellar instability associated with patellofemoral dysplasia: a report of 26 cases. Orthop Traumatol Surg Res. 2012;98(2):167-72.

11. Frosch KH, Schmeling A. A new classification system of patellar instability and patellar maltracking. Arch Orthop Trauma Surg. 2016;136(4):485-97.

12. Tscholl PM, Wanivenhaus F, Fucentese SF. Conventional Radiographs and Magnetic Resonance Imaging for the Analysis of Trochlear Dysplasia: The Influence of Selected Levels on Magnetic Resonance Imaging. Am J Sports Med. 2017;45(5):1059-65.

13. Carrillon Y, Abidi H, Dejour D, Fantino O, Moyen B, Tran-Minh VA. Patellar instability: assessment on MR images by measuring the lateral trochlear inclination-initial experience. Radiology. 2000;216(2):582-5.

14. Pennock AT, Alam M, Bastrom T. Variation in tibial tubercle-trochlear groove measurement as a function of age, sex, size, and patellar instability. Am J Sports Med. 2014;42(2):389-93.

15. Lee PP, Chalian M, Carrino JA, Eng J, Chhabra A. Multimodality correlations of patellar height measurement on X-ray, CT, and MRI. Skeletal Radiol. 2012;41(10):1309-14.

16. Tscholl PM BD, Fucentese SF. Validation of patellar height measurements on conventional radiographs and MRI in patients with patellar instability. EFFORT congress 2017; Vienna2017.

17. Biedert RM, Albrecht S. The patellotrochlear index: a new index for assessing patellar height. Knee Surg Sports Traumatol Arthrosc. 2006;14(8):707-12. 
18. Dejour D, Ferrua P, Ntagiopoulos PG, Radier C, Hulet C, Remy F, et al. The introduction of a new MRI index to evaluate sagittal patellofemoral engagement. Orthop Traumatol Surg Res. 2013;99(8 Suppl):S391-8.

19. Munch JL, Sullivan JP, Nguyen JT, Mintz D, Green DW, Shubin Stein BE, et al. Patellar Articular Overlap on MRI Is a Simple Alternative to Conventional Measurements of Patellar Height. Orthop J Sports Med. 2016;4(7):2325967116656328.

20. Diederichs G, Issever AS, Scheffler S. MR imaging of patellar instability: injury patterns and assessment of risk factors. Radiographics. 2010;30(4):961-81.

21. Tscholl PM, Antoniadis A, Dietrich TJ, Koch PP, Fucentese SF. The tibial-tubercle trochlear groove distance in patients with trochlear dysplasia: the influence of the proximally flat trochlea. Knee Surg Sports Traumatol Arthrosc. 2016;24(9):2741-7.

22. Grelsamer RP, Saleh J, Gladstone J. Congruous versus incongruous patellar tilt-a preliminary study. Bull NYU Hosp Jt Dis. 2012;70(4): 232-4.

23. Diederichs G, Kohlitz T, Kornaropoulos E, Heller MO, Vollnberg B, Scheffler S. Magnetic resonance imaging analysis of rotational alignment in patients with patellar dislocations. Am J Sports Med. 2013;41(1):51-7.

24. Reikeras O. Patellofemoral characteristics in patients with increased femoral anteversion. Skeletal Radiol. 1992;21(5):311-3.

25. Billing L. Roentgen examination of the proximal femur end in children and adolescents; a standardized technique also suitable for determination of the collum-, anteversion-, and epiphyseal angles; a study of slipped epiphysis and coxa plana. Acta Radiol Suppl. 1954;110:1-80.

26. Fabry G, MacEwen GD, Shands AR, Jr. Torsion of the femur. A follow-up study in normal and abnormal conditions. J Bone Joint Surg Am. 1973;55(8):1726-38.

27. Sutter R, Dietrich TJ, Zingg PO, Pfirrmann CW. Femoral antetorsion: comparing asymptomatic volunteers and patients with femoroacetabular impingement. Radiology. 2012;263(2):475-83.

28. Sutter R, Dietrich TJ, Zingg PO, Pfirrmann CW. Assessment of Femoral Antetorsion With MRI: Comparison of Oblique Measurements to Standard Transverse Measurements. AJR Am J Roentgenol. 2015;205(1):130-5.

29. Tscholl PM, Biedert RM, Wanivenhaus F, Fucentese SF. Patellar tendinopathy with intratendinous alteration on MRI may be related to patellofemoral dysplasia. Scand J Med Sci Sports. 2018;28(4):1443-50.

30. Campagna R, Pessis E, Biau DJ, Guerini H, Feydy A, Thevenin FS, et al. Is superolateral Hoffa fat pad edema a consequence of impingement between lateral femoral condyle and patellar ligament? Radiology. 2012;263(2):469-74.

31. Ciriello V, Gudipati S, Tosounidis T, Soucacos PN, Giannoudis PV. Clinical outcomes after repair of quadriceps tendon rupture: a systematic review. Injury. 2012;43(11):1931-8. 